\title{
Actividad antimicrobiana de propóleos de abejas sin aguijón en combinación con ajo, Allium sativum (Amaryllidaceae)
}

\author{
Julieta Grajales Conesa ${ }^{1}$, Julián Elías Chirino¹, Eduardo Lozano Guzmán², \\ Francisco Moreno Cruz ${ }^{2}$, Víctor Albores Flores ${ }^{1 *} \&$ Alfonso López García ${ }^{1}$ \\ 1. Instituto de Biociencias (IBC), Universidad Autónoma de Chiapas. Blvd. Príncipe Akishino s/n, Col. Solidaridad \\ 2000. Tapachula Chiapas, México; jugrajco79@gmail.com, javierelias_c@hotmail.com, alboresflores@gmail.com, \\ josealoga@hotmail.com \\ 2. Laboratorio de Farmacognosia, Facultad de Ciencias Químicas, Universidad Juárez del Estado de Durango, Av. \\ Veterinaria s/n, Circuito Universitario, Col. Valle del Sur, Durango, México; elozano@ujed.mx; jmoreno@ujed.mx \\ * Correspondence
}

Recibido 27-III-2020. Corregido 11-VIII-2020. Aceptado 30-IX-2020.

\begin{abstract}
Stingless bees propolis antimicrobial activity in combination with garlic, Allium sativum (Amaryllidaceae). Introduction: The excessive use of antibiotics has increased pathogenic microorganisms resistance, which derives in patient mortality. Therefore, the strategies for searching new natural and unconventional strategies has become constant and important. Objective: To determine the antimicrobial activity against methicillin-resistant Staphylococcus aureus (MRSA) against stingless bees propolis. Methods: We evaluated nine (20\%) propolis from stingless bees ethanolic extracts from different regions: Melipona beecheii, Melipona solani, Tetragonisca angustula and Scaptotrigona mexicana. The chemical characterization was performed by liquid chromatography (HPLC) and microbial resistance tests by the macrodilution method, to determine the effect of the combinations. Results: The compounds that were of interests and the most abundant were the following; hydroxycinnamic acids, flavanones, flavonoids and the glycosylated derivatives. Four of the nine propolis were effective against MRSA, which came from the following species; one from $M$. solani and three from $S$. mexicana. The minimum inhibitory concentration for $S$. mexicana was in the range of 3-8 mg mL-1 and for M. solani it was $4 \mathrm{mg} \mathrm{mL-1}$. Isobolographic studies resulted in an additive effect $(\gamma=1)$ for the combination of Allium sativum with the $S$. mexicana propolis sambles and an antagonistic effect $(r>1)$ for the combination of A. sativum with the propolis of $M$. solani. Conclusions: the combination of extracts with lower concentrations of A. sativum, may be the most effective, than those that were individually tested. More detailed studies are required to define the mechanisms of stingless bees propolis as well as their combination with other organic substances.
\end{abstract}

Key words: extracts; Meliponini; stingless bees; Staphylococcus aureus; isobolograms; inhibition.

Grajales Conesa, J., Elías Chirino, J., Lozano Guzmán, E., Albores Flores, V., \& López García, A. (2021). Actividad antimicrobiana de propóleos de abejas sin aguijón en combinación con ajo, Allium sativum (Amaryllidaceae). Revista de Biología Tropical, 69(1), 23-35. DOI 10.15517/rbt.v69i1.41241

Hasta el 2014, las principales causas de morbilidad han sido las enfermedades infecciosas, los tres primeros lugares los han ocupado las infecciones respiratorias agudas, las infecciones intestinales por otros organismos y las de vías urinarias (Soto, Moreno, \& Pahua, 2014). El uso excesivo de antibióticos para tratar estas infecciones ha traído el aumento de resistencia de los microorganismos reduciendo las posibilidades de tratamientos eficaces y aumento en el riesgo de mortalidad de los pacientes (Andersson \& Hughes, 2011; Serra, 2017). Por lo consiguiente, la búsqueda de nuevas estrategias naturales y no convencionales 
se ha vuelto constante (Morroni et al., 2018). Estudios recientes han demostrado que los propóleos son una fuente de compuestos bioactivos con distintas actividades; antibacterial, fungistática, antiviral, antioxidante, antiinflamatoria, citotóxica y anticancerígena (Sartori et al., 2012, Popova, Chinou, Marekov, \& Bankova, 2013, Choudhari, Haghniaz, Rajwade, \& Paknikar, 2013, Inui et al., 2014, Wang et al., 2014, Nina et al., 2015).

Los propóleos es una substancia que las abejas utilizan para construir y proteger la colmena. Es elaborado a partir de savias, resinas, gomas y mucílagos de plantas mezcladas con cera y secreciones bucales (Massaro, Simpson, Powell, \& Brooks, 2015). Se ha encontrado que algunas especies de abejas neotropicales recolectan barro para agregarlo a la mezcla (Araújo et al., 2015). Por lo tanto, la zona geográfica y las plantas de origen determinarán la composición química de los propóleos (Santos et al., 2017). En diversos estudios enfocados en su caracterización química, se han reportado aproximadamente 300 compuestos, destacando los flavonoides, terpenos y fenoles (Huang, Zhang, Wang, Li, \& Hu, 2014).

Por otro lado, existen otras fuentes naturales para la obtención de compuestos bioactivos que también pudieran combatir resistencias microbianas como es el caso de ajo (Allium sativum) y otras plantas de la familia Allium. La alicina, alina y otros compuestos ricos en azufre, incluyendo compuestos fenólicos de alta reactividad, son componentes principales de A. sativum con propiedades antibacterianas (Guillamón, 2018).

Una de las posibilidades para mejorar el espectro de acción de las terapias actuales es el uso combinado de antibióticos, convencionales y no convencionales, con fuentes naturales (Amber, Aijaz, Immaculata, Luqman, \& Nikhat, 2010, Chanda \& Rakholiya, 2013, Ushimaru, Barbosa, Fernández, Stasi, \& Júnior, 2012, Lozano et al., 2013).

En ensayos in vitro con Staphylococcus aureus meticilina resistente (SARM), en la combinación de extracto de propóleos de Apis mellifera con ajo (Allium sativum) se han encontrado efectos inhibitorios sinérgicos (Rajic-Savic \& Valovic; 2009, Moreno-Cruz et al., 2014).

Sin embargo, dado que no hay reportes de estudios del efecto que tienen los propóleos producidos por abejas sin aguijón en combinación con ajo sobre SARM, el presente estudio tiene como objetivo evaluar la actividad antimicrobiana de propóleos producidos por abejas sin aguijón en combinación con extractos de ajo (Allium sativum), específicamente contra Staphylococcus aureus resistente a meticilina (SARM).

\section{MATERIALES Y MÉTODOS}

Propóleos: Se obtuvieron nueve muestras de propóleos a través de la "Asociación de Meliponicultores del Soconusco S.C. de R. L.", ubicados en los municipios de la región del Soconusco en: Tapachula (Rancho "San Juan" Viva México (RSJ): (1454'00" N \& 92 $19^{\circ}$ '01" $\mathrm{O})$, Cantón la pita rancho agroecológico "Ayol" (TAP) $\left(14^{\circ} 49^{\prime} 46^{\prime \prime} \mathrm{N} \& 92^{\circ} 17^{\prime} 42^{\prime}\right.$ 'W), Tuxtla Chico (Izapa I; TCIa e Izapa II; TCIb) (145'19" N \& 92 $11^{\circ} 19$ ” W), Cacahoatán (CAC): (150'13” N \& 92¹0'6”'W) y Mazatán (MAZ): Rancho "El cocal II" (1450'22.2" N \& 92 10 '58.6” W).

Las especies de abejas sin aguijón y el sitio de muestreo fueron los siguientes: Melipona beecheii (MbTAP, MbTCIa), M. solani (MsTCIa), Tetragonisca angustula (TaRSJ) y Scaptotrigona mexicana (SmTAP, SmRSJ, SmCAC, SmMAZ, SmTCIb). Las muestras se colectaron con herramientas de acero inoxidable previamente esterilizadas, tomando aproximadamente $50 \mathrm{~g}$ de propóleos. Las muestras fueron trasladadas al laboratorio y conservadas en refrigeración a $4-8^{\circ} \mathrm{C}$.

Extractos: A todas las muestras de propóleos se les retiraron, manualmente, los residuos de plantas, insectos y trozos de maderas desprendidos de la caja al momento de la recolecta. Se pesaron $30 \mathrm{~g}$ de propóleos y se depositaron en frascos estériles protegidos de la luz con $100 \mathrm{~mL}$ de etanol grado HPLC (Fermont $\left.^{\mathbb{R}}\right)(99.5 \%)$. Se agitaron manualmente 
por un lapso de 15 min durante 8 días. Posteriormente, el contenido de los frascos se filtró a través de papel Whatman No. 41. Para la elaboración de los extractos de ajo, los frutos se adquirieron en el mercado local. Una vez retirada la cáscara fueron cortados en trozos de $1 \mathrm{~cm}$. Se pesaron $30 \mathrm{~g}$ de ajo y fueron depositados en frascos protegidos de la luz con 100 $\mathrm{mL}$ de etanol $(99.5 \%)$ por ocho días. Luego fueron filtrados tal como se describe para los propóleos. Todos los extractos se almacenaron en refrigeración a 4-8 ${ }^{\circ} \mathrm{C}$ (Lozano et al., 2013, Moreno et al., 2014).

Caracterización química: La caracterización química de los propóleos se determinó por medio de Cromatografía Líquida de Alta Resolución en un equipo Agilent 1200 (con bomba binaria y autoinyector). Las condiciones de operación fueron: temperatura de columna $25^{\circ} \mathrm{C}$, flujo de fase móvil de $1 \mathrm{~mL} / \mathrm{min}$, en relación 35:65 v/v. Volumen de inyección 2 $\mu \mathrm{L}$. Fragmentador a $135 \mathrm{~V}$. Modo Scan. Rango de masas de 100 a 1000 . Polaridad positiva y negativa. Modo de ionización electroespray ESI. Temperatura de gas secante a $200{ }^{\circ} \mathrm{C}$. Flujo de gas de $13 \mathrm{~L} / \mathrm{min}$ y nebulizador a $2 \mathrm{~atm}$. Tiempo de corrida de $20 \mathrm{~min}$. Cada $5 \mathrm{~min}$ se realizó la lectura correspondiente para obtener los compuestos de los propóleos hasta completar una corrida total de $20 \mathrm{~min}$.

Preparación del inóculo: Se utilizaron cultivos de SARM provenientes del laboratorio de la Facultad de Ciencias Químicas de la UJED previamente caracterizadas con base a lo establecido por el Clinical and Laboratory Standards Institute (2013). Al momento del estudio, las cepas se encontraban preservadas a $-20{ }^{\circ} \mathrm{C}$ en medio Skim Milk. Las cepas se activaron resembrándolas en caldo MuellerHinton durante $24 \mathrm{~h}$ a $35^{\circ} \mathrm{C}$. Posteriormente se sembró por extensión en placas de Petri con agar Mueller-Hinton adicionadas con $2 \%$ de $\mathrm{NaCl}$ para aumentar la selectividad del medio y se incubaron por $24 \mathrm{~h}$ a $35^{\circ} \mathrm{C}$. Finalmente, se tomaron cinco colonias y se suspendieron en $100 \mathrm{~mL}$ de caldo Mueller-Hinton adicionado con $\mathrm{NaCl}$ al $2 \%$. La determinación de la concentración celular se realizó por conteo al microscopio en cámara de Neubauer.

Estudio de inhibición: Se siguió el método de macrodilución (Taroco, Seija, \& Vignoli, 2006). Con base a trabajos previos (Lozano et al., 2013; Moreno et al., 2014), se ensayaron seis rangos de dilución: 1:4, 1:8, 1:16, 1:32, 1:64 y $1: 128(\mathrm{v} / \mathrm{v})$ para los extractos de propóleos y para el extracto de ajo se ensayaron siete rangos de dilución: 1:2, 1:4, 1:8, 1:16, 1:32, 1:64 y 1:128 (v/v). Se depositó $1 \mathrm{~mL}$ de caldo Mueller-Hilton estéril como diluyente en cada tubo. Posteriormente se depositó $1 \mathrm{~mL}$ del extracto correspondiente en el primer tubo de la serie, se agitó y se transfirió $1 \mathrm{~mL}$ al siguiente tubo hasta llegar al sexto tubo. Al último tubo de la serie se le extrajo $1 \mathrm{~mL}$ y se desechó. Al final, se depositó $1 \mathrm{~mL}$ de inoculo en cada tubo para lograr las concentraciones de los extractos antes mencionadas. Se agitaron los tubos y se dejaron reposar durante $20 \mathrm{~min}$ para que el extracto funcionara. Posterior a esto, se sembraron $10 \mu \mathrm{L}$ de cada tubo en placas con agar Mueller-Hinton adicionadas con $\mathrm{NaCl}$ al $2 \%$. Tanto los tubos como las placas. Para descartar el efecto inhibitorio del etanol, se realizó el mismo proceso sustituyendo tanto los extractos de propóleos como de ajo, por etanol. Todas las pruebas se hicieron por triplicado. Después del tiempo de incubación se contabilizaron las colonias crecidas en placas para determinar la población expresada como UFC x $10^{6}$.

Concentración mínima inhibitoria, concentración mínima bactericida y concentración efectiva 50: Para determinar la Concentración Mínima Inhibitoria (CMI) se leyó la absorbancia a los tubos inoculados por espectrometría a una longitud de onda de 625 $\mathrm{nm}$, usando como "blanco" caldo estéril. Esto se realizó para cada una de las repeticiones.

Por otra parte, se realizó el conteo de colonias en placa y se reportó como UFCx $10^{6} \mathrm{~mL}^{-1}$. Se estableció como Concentración Mínima Bactericida (CMB) aquella concentración a la cual no hubiera crecimiento en placa. 
La concentración efectiva 50 (CE50) se calculó de la siguiente forma: a) en cada caso se determinó la mortalidad como la diferencia entre la población inicial (inóculo) y la contabilizada en la placa a cada concentración de antibiótico. b) se denominó al porcentaje de efecto (PE) como la relación entre la media de la mortalidad para las tres corridas en cada concentración y el inóculo expresado en porcentaje. c) Para obtener la curva dosisrespuesta se realizó un gráfico del log de las concentraciones en el eje de las abscisas y el $\mathrm{PE}$ en el eje de las ordenadas obteniéndose una recta. Con ello, se determinó la ecuación de la recta correspondiente. d) Finalmente, con dicha ecuación se calculó la concentración a la cual se obtendría el $50 \%$ de efecto (CE50). Para expresar las CMI, CMB Y CE50 en $\mathrm{mg} \mathrm{mL}$ ${ }^{-1}$, se colocaron $5 \mathrm{~mL}$ de cada extracto en una termobalanza a $36{ }^{\circ} \mathrm{C}$ durante $90 \mathrm{~min}$. Se realizaron lecturas cada 10 min hasta observar que ya no había cambios en el peso. Esto se realizó por triplicado.

Análisis de datos: Las medias de la concentración mínima inhibitoria (CMI) para la combinación de extractos de propóleos y ajo, y concentración mínima bactericida (CMB) de los diferentes tratamientos fueron sometidos a un ANOVA para determinar diferencia entre las medias de los tratamientos y en los que hubo diferencia se realizó un contraste de dichas medias con prueba Tukey a través del Software estadístico Infostat 2015.

Combinaciones: Se utilizó la técnica para estudios isobolográficos descrita por Tallarida en 2001. La línea se estableció con base a la $\mathrm{CE}_{50}$ promedio de la cepa SARM, como se describió líneas arriba. Según la técnica citada, al combinar un antimicrobiano A, la dosis de 50 $\%$ (CE50 - punto A en la Fig. 1) con una dosis igual de $50 \%$ de otro antimicrobiano B (punto B), se obtiene un efecto conjunto del $100 \%$, es decir, equivalente al obtenido por cualquiera de ellos si se administrara de forma independiente. La línea que une ambos puntos se denomina "línea de aditividad" o "isobola" (Fig. 1).

Para evaluar un posible efecto sinérgico se ensayaron las combinaciones descritas en la Tabla 1. Con los resultados obtenidos, se determinaron las CE50e de las mezclas, así como las concentraciones individuales (Tabla 2). Las medias de las CE50 calculadas se compararon

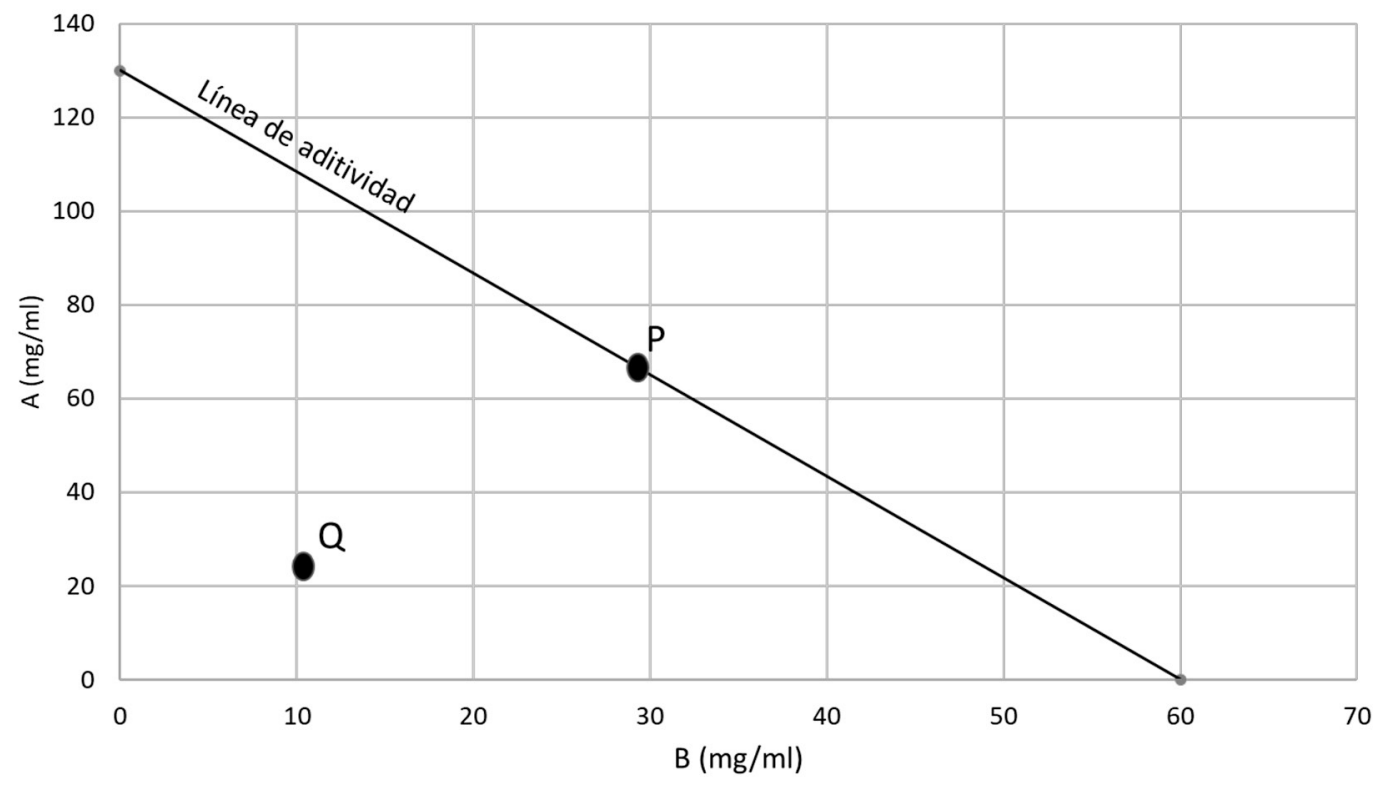

Fig. 1. Isobolograma, línea de aditividad. / Fig. 1. Isobologram, additivity line. 
TABLA 1

Combinaciones teóricas usadas en el estudio Isobolográfico expresados en $\mathrm{mg} \mathrm{mL}^{-1}$

TABLE 1

Theoretical combinations used in the Isobolographic study expressed in $\mathrm{mg} \mathrm{mL}^{-1}$

\begin{tabular}{|c|c|c|c|c|c|c|c|c|c|c|c|}
\hline \multirow{2}{*}{\multicolumn{2}{|c|}{$\begin{array}{l}\text { A.s. SmRSJ } \\
\text { Combinación }\end{array}$}} & \multirow{3}{*}{$\mathrm{zt}$} & A.s. & \multicolumn{2}{|c|}{ SmMAZ } & A.s. & \multicolumn{2}{|c|}{$\mathrm{SmTCIb}$} & A.s. & \multicolumn{2}{|c|}{ MsTCla } \\
\hline & & & \multicolumn{2}{|c|}{ Combinación } & \multirow[t]{2}{*}{$\mathrm{zt}$} & \multicolumn{2}{|c|}{ Combinación } & \multirow[t]{2}{*}{$\mathrm{zt}$} & \multicolumn{2}{|c|}{ Combinación } & $\mathrm{zt}$ \\
\hline 4.2 & 1.7 & & 4.2 & 2.7 & & 4.2 & 1 & & 4.2 & 1.40 & \\
\hline 2.1 & 0.85 & 2.95 & 2.1 & 1.35 & 3.45 & 2.1 & 0.5 & 2.6 & 2.1 & 0.70 & 2.8 \\
\hline 1.05 & 0.425 & 1.47 & 1.05 & 0.33 & 1.72 & 1.05 & 0.25 & 1.3 & 1.05 & 0.35 & 1.4 \\
\hline
\end{tabular}

A.s.: Allium sativum; zt: suma de ambos extractos. zt: sum of both extracts.

TABLA 2

Combinaciones experimentales expresadas en $\mathrm{mg} \mathrm{mL}^{-1}$ e índice de interacción $\gamma$

TABLE 2

Experimental combinations expressed in $\mathrm{mg} \mathrm{mL}^{-1}$ and interaction index $\gamma$

\begin{tabular}{|c|c|c|c|c|c|c|c|c|c|}
\hline A.s. & SmRSJ & & & & A.s. & SmMAZ & & & \\
\hline CE50 & & Zmix & $\mathrm{Zt}$ & $\gamma$ & CE50 & & Zmix & $\mathrm{Zt}$ & $\gamma$ \\
\hline 0.0021 & 0.0008 & 0.0029 & 2.95 & 1 & 1.0174 & 1.5826 & 2.6 & 3.45 & 1 \\
\hline A.s. & $\mathrm{SmTClb}$ & & & & A.s. & MsTCla & & & \\
\hline CE50 & & Zmix & $\mathrm{zt}$ & $\gamma$ & CE50 & & Zmix & $\mathrm{Zt}$ & $r$ \\
\hline 0.0113 & 0.0027 & 0.014 & 2.6 & 1 & 4.6385 & 1.5615 & 6.2 & 2.8 & 0.3 \\
\hline
\end{tabular}

A.s.: Allium sativum.

Zmix: Combinación efectiva 50 experimental; Zt: Combinación efectiva 50 teórica; Índice de interacción $(\gamma): \gamma=1$, efecto aditivo, $\gamma<1$, efecto sinérgico y $\gamma>1$, efecto antagónico.

Zmix: Experimental effective combination 50; Zt: Theoretical 50 effective combination; Interaction index $(\gamma): \gamma=1$, additive effect, $\gamma<1$, synergistic effect and $\gamma>1$, antagonistic effect.

con las CE50e para verificar si fueron diferentes mediante la prueba " $t$ " Student para datos independientes. Posteriormente se calculó el índice de interacción $(\gamma)$ con estos datos, mediante la siguiente ecuación:

$$
r=a / A+b / B
$$

Donde: $\mathrm{A}=$ Concentración de propóleos administrado en forma individual que presenta el $100 \%$ de inhibición.

$\mathrm{B}=$ Concentración de Allium sativum administrado en forma individual que presenta el 100 $\%$ de inhibición.

a y $b=$ Concentraciones de cada antimicrobiano usadas en la combinación

Si $\gamma$ es igual a 1 , se tiene un efecto simple aditivo, si es mayor que 1 el efecto se considera antagónico. Finalmente, si $\gamma$ es menor que 1 y la prueba " $t$ " arroja diferencias significativas, se considera un efecto sinérgico.

\section{RESULTADOS}

\section{Caracterización química de los propó-} leos: Los resultados de análisis por HPLC/MS/ MS de los distintos propóleos se muestran en la Tabla 3. Se tomó como ion de referencia el ácido ferúlico debido a que presentó la mayor abundancia iónica. Los propóleos de abejas sin aguijón llegaron a contener alrededor de 16 compuestos, los cuales pertenecen a los ácidos orgánicos y derivados de los terpenos, y se presentaron en mayor proporción respecto al ion de referencia. El ácido ferúlico fue identificado en todas las muestras, mientras 
TABLA 3

Compuestos identificados en las nueve muestras de propóleos a través de HPLC-QQQ/MS/MS

TABLE 3

Compounds identified in the nine propolis samples via HPLC-QQQ / MS / MS

\begin{tabular}{|c|c|c|c|c|}
\hline Grupo & Masa & $\mathrm{IP} \mathrm{m} / \mathrm{z}^{1}$ & Compuesto & Ocurrencia $^{2}$ \\
\hline \multicolumn{5}{|l|}{ ÁCIDO } \\
\hline 1 & 149 & $(-) 148.8$ & Ácido cinámico; Ácido (E)-3-fenil-2-propenoico & 9 \\
\hline 2 & 181 & 181 & Ácido cafeíco; Ácido (E)-3-(3,4-dihidroxifenil)prop-2-enoico & $2,8,9$ \\
\hline 3 & 193 & $(+) 194.20$ & $\begin{array}{l}\text { Ácido ferúlico; Ácido (E)-3-(4-hidroxi-3-metoxi-fenil)propil- } \\
\text { 2-enoico }\end{array}$ & $1,2,3,4,6,7,8,9$ \\
\hline \multicolumn{5}{|c|}{ CUMARINA } \\
\hline 4 & 244 & 244.29 & Suberosina; 7-Metoxi-6- (3-metilbut-2-enil) cromen-2-ona & 4,6 \\
\hline \multicolumn{5}{|c|}{ FLAVONOIDE } \\
\hline 5 & 253 & $(+) 254.20$ & Crisina; 5,7-Dihidroxi-2-fenil-4H-cromen-4-ona & 9 \\
\hline 6 & 422 & 422.47 & $\begin{array}{l}\text { Macarangina; 6-[(2E)-3,7-dimetilocta-2,6-dienil]-3,5,7- } \\
\text { trihidroxi-2-(4-hidroxifenil)cromen-4-ona }\end{array}$ & $4,7,8,9$ \\
\hline \multicolumn{5}{|c|}{ FLAVANONA } \\
\hline 7 & 255 & 255.30 & $\begin{array}{l}\text { Pinocembrina; 5,7-Dihidroxi-2-fenil-2,3-dihidro-4H-cromen- } \\
\text { 4-ona }\end{array}$ & $2,3,6,8,9$ \\
\hline 8 & 272 & 272.20 & Naringenina; 5,7-Dihidroxi-2-(4 hidroxifenill)croman-4-ona & $2,3,5,6,7,8,9$ \\
\hline \multicolumn{5}{|c|}{ 年 } \\
\hline 9 & 269 & $(+) 271.8$ & Apigenina; 5,7-dihidroxi-2-(4-hidroxifenil)cromen-4-ona & 3,9 \\
\hline \multicolumn{5}{|c|}{ DITERPENO } \\
\hline 10 & 301 & $(-) 300.70$ & $\begin{array}{l}\text { Totarolona; 7-hidroxi-1,1,4a-trimetil-8-propan-2-il- } \\
\text { 4,9,10,10a-tetrahidro-3H-fenantren-2-ona }\end{array}$ & $1,2,5,7,8,9$ \\
\hline \multicolumn{5}{|c|}{ TERPENOIDE } \\
\hline 11 & 314 & 314.29 & $\begin{array}{l}\text { Odoratina; 7-hidroxi-3-(3-hidroxi-4-metoxifenil)-6- } \\
\text { metoxicromen-4-ona }\end{array}$ & $4,8,9$ \\
\hline \multicolumn{5}{|c|}{ NO IDENTIFICADO } \\
\hline 12 & & 550.3 & & $1,3,4,6,7,8,9$ \\
\hline 13 & & 633.1 & & $1,3,4,6,7,8,9$ \\
\hline 14 & & 702.5 & & $2,3,6,9$ \\
\hline 15 & & 727.70 & & $1,3,4,6,7,8,9$ \\
\hline 16 & & 734.70 & & $1,3,4,6,7,8,9$ \\
\hline
\end{tabular}

1. Iones principales $\mathrm{m} / \mathrm{z}$ : positivo $[\mathrm{M}+\mathrm{H}]^{+}$y negativo $[\mathrm{M}+\mathrm{H}]^{-} ;{ }^{2}$ Presencia: Los números representan la presencia del compuesto en los extractos de propóleos. Las claves son las siguientes; MbTAP (1), MbTCIa (2), MsTCla (3), TaRSJ (4), SmTAP (5), SmRSJ (6), SmCAC (7), SmMAZ (8) y SmTCIb (9).

1. Principal $\mathrm{m} / \mathrm{z}$ ions: positive $[\mathrm{M}+\mathrm{H}]+$ and negative $[\mathrm{M}+\mathrm{H}]-;{ }^{2}$ Occurrence: The numbers represent the presence of the compound in the propolis extracts. The keys are as follows: MbTAP (1), MbTCIa (2), MsTCla (3), TaRSJ (4), SmTAP (5), SmRSJ (6), SmCAC (7), SmMAZ (8) y SmTCIb (9).

que la naringenina y totarolona fueron detectados en siete de las nueve muestras recolectadas.

Estudio de inhibición: Los resultados muestran que cuatro de los nueve extractos evaluados contra SARM fueron eficaces para inhibir el crecimiento de la bacteria durante las $24 \mathrm{~h}$ de la prueba (Tabla 4). Estos extractos pertenecen a las abejas sin aguijón Melipona solani (MsTCla) y Scaptotrigona mexicana (SmRSJ, SmMAZ y SmTCIb). El análisis estadístico no mostró diferencias entre los cuatro propóleos que resultaron positivos para inhibir a SARM $(\mathrm{P}>0.05)$. 
TABLA 4

Porcentaje de inhibición de los extractos de propóleos contra Staphylococcus aureus resistente a meticilina (SARM), concentración mínima inhibitoria (CMI) y concentración efectiva 50 (CE50) expresada en mg mL-1

TABLE 4

Inhibition percentage of propolis extracts against methicillin-resistant Staphylococcus aureus (MRSA), minimum inhibitory concentration (MIC) and effective concentration 50 (EC50) expressed in $\mathrm{mg} \mathrm{mL}-1$

\begin{tabular}{ccccccccccc} 
[Ext] & MbTAP & MbTCIa & TaRSJ & SmTAP & SmCAC & MsTCla & SmRSJ & SmMAZ & SmTCIb & As \\
$20 \%$ & $\mathrm{X}$ & $\mathrm{X}$ & $\mathrm{X}$ & $\mathrm{X}$ & $\mathrm{X}$ & 100 & 100 & 100 & 100 & \\
$\mathrm{CMI}$ & & & & & & 4 & 5 & 8 & 3 & 9 \\
$\mathrm{CE} 50$ & & & & & & 1.41 & 1.7 & 2.7 & 1 & 4.2 \\
\hline
\end{tabular}

La "X" representa que no hubo inhibición contra SARM; [Ext]: Concentración del extracto; As: Allium sativum; M. solani (MsTCla) y S. mexicana (SmMAZ, SmTClb, SmTClb).

The "X" represents that there was no inhibition against MRSA; [Ext]: Concentration of the extract; As: Allium sativum; M. solani (MsTCla) and S. mexicana (SmMAZ, SmTClb, SmTClb).

Todos los propóleos a la dilución 1:4 tuvieron una concentración mínima inhibitoria (CMI) de $4 \mathrm{mg} \mathrm{mL}^{-1}$ (MsTCla), $5 \mathrm{mg}$ $\mathrm{mL}^{-1}$ (SmRSJ), $8 \mathrm{mg} \mathrm{mL}^{-1}$ (SmMAZ) y $3 \mathrm{mg}$ $\mathrm{mL}^{-1}$ (SmTClb). La concentración efectiva 50 (CE50) fue de 1.41, 1.7, 2.7 y $1 \mathrm{mg} \mathrm{mL}^{-1}$ respectivamente. En el caso de Allium sativum, se registró una CMI de $9 \mathrm{mg} \mathrm{mL}^{-1}$ y la CE50 de $4.2 \mathrm{mg} \mathrm{mL}^{-1}$ (Tabla 4) de acuerdo a lo descrito en la sección de materiales y métodos.
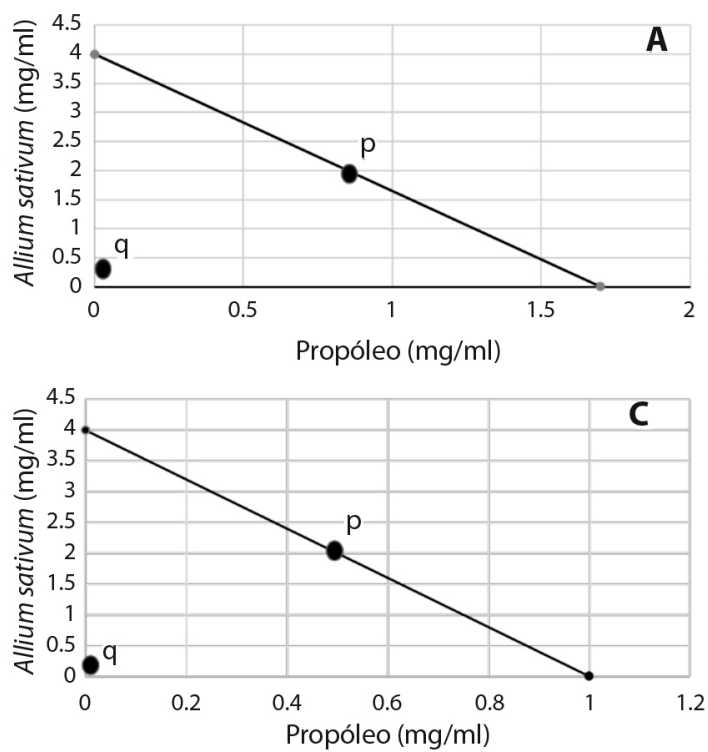

Combinaciones: Como se observa en los isobologramas (Fig. 2), para los cuatro extractos existe diferencia entre la combinación efectiva 50 teórica (CE50t) y la experimental (CE50e). El punto P representa la CE50t mientras que el punto Q representa la CE50e. Para el caso de la CE50e de los extractos de SmRSJ, SmMAZ, SmTClb, esta se encuentra por debajo de la línea de aditividad lo que significaría
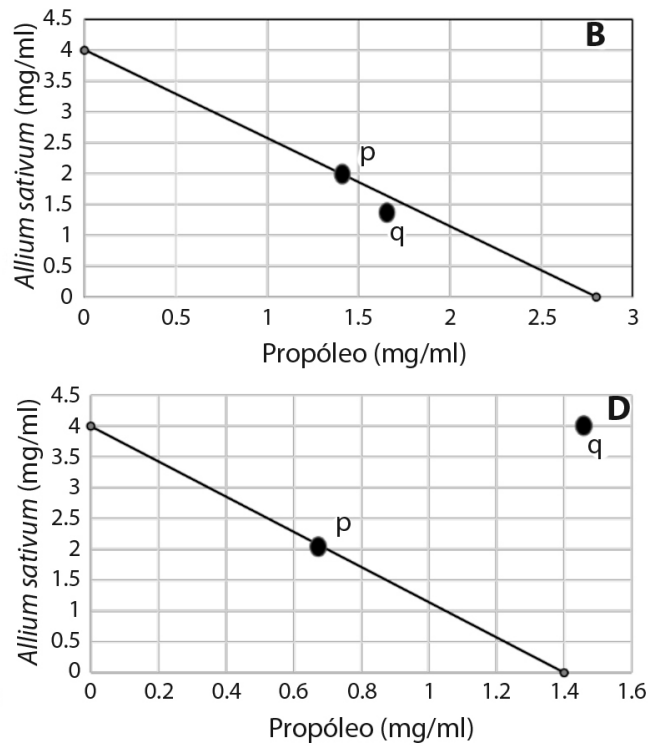

Fig. 2. Isobologramas de los extractos de propóleos. Punto rojo: Concentración efectiva 50 teórica (zt). Punto negro: Concentración efectiva experimental (zmix).

Fig. 2. Isobolograms of propolis extracts. Red dot: Theoretical effective concentration 50 (zt). Black point: Experimental effective concentration (zmix). 
que la combinación de ambos extractos. Sin embargo, la CE50e de MsTCla se encontró por encima de la línea de aditividad, esto significaría que la combinación de ambos extractos produciría un efecto adverso en comparación de la aplicación individual tanto del propóleos como de Allium sativum.

\section{DISCUSIÓN}

Los compuestos encontrados en los propóleos de abejas sin aguijón han sido reportados con actividad antimicrobiana y descritos mayoritariamente para propóleos de Apis mellifera (Bankova, De Castro, \& Marcucci, 2000; Marcucci et al., 2001; Ristivojević et al., 2016).

El ácido cinámico, cafeíco y ferúlico se encuentran como constituyentes habituales en la composición química de los propóleos, principalmente los que se producen en regiones tropicales como Brasil (Dos Santos, Bicalho, \& de Aquino Neto, 2003; Salomão et al., 2004; Sawaya, Souza, Marcucci, Cunha, \& Shimizu, 2004). Además de estos ácidos aromáticos, también se encuentran los flavonoides y sus derivados tales como la crisina, macarangina, naringenina, pinocembrina y apigenina, compuestos recurrentes en la mayoría de los propóleos de A. mellifera y las abejas sin aguijón (Silici \& Kutluca, 2003, Ahn, Kumazawa, Hamasaka, Bang, \& Nakayama, 2004, Kumazawa, Hamasaka, \& Nakayama, 2004, Lotti et al., 2010).

Los terpenos y sus derivados son otra clase de compuestos que comúnmente se encuentran dentro del perfil químico. La totarolona y odoratina han sido reportados en propóleos griegos (Popova, Dimitrova, Al-Lawati, Tsvetkova, Najdenski, \& Bankova, 2009) y de Népal (Shrestha, Narukawa, \& Takeda, 2007). Estos resultados concuerdan con los obtenidos en este trabajo, ya que la mayoría de los propóleos cuentan con la presencia de uno o más compuestos similares. Sin embargo, la relación en la que están dentro de la composición química de cada propóleos resulta en características específicas para cada uno (Bonvehí \& Coll, 1994). Del compuesto doce al dieciséis no lograron ser identificados, sin embargo, se encuentran en la mayoría de todos los extractos, por lo que la identificación podría llevarse a cabo en futuras investigaciones. La suberosina, una lactona del ácido hidroxicinámico, ha sido reportada en propóleos de zonas como Argelia (Boutabet, Kebsa, Alyane, \& Lahouel, 2011), Brasil (De Castro, 2001) e Irán (Trusheva et al., 2010).

Los valores de CMI encontrados en este estudio son similares a los reportados por otros autores, tanto para propóleos de A. mellifera como para las abejas sin aguijón. Estos valores van desde los $0.0025 \mathrm{mg} \mathrm{mL}^{-1}$ hasta los $9 \mathrm{mg}$ $\mathrm{mL}^{-1}$ (Fernandes, Leomil, Fernades, \& Sforcin, 2001, Miorin, Levy Junior, Custodio, Bretz \& Marcucci, 2003, Castro et al., 2009). Tal es el caso del estudio realizado con $M$. orbignyi donde reportan una CMI de $3.1 \mathrm{mg} \mathrm{mL}^{-1}$ contra S. aureus (Campos et al., 2014), este resultado es similar a lo encontrado en el presente estudio y que además se encuentra dentro del rango de concentración reportado para otras especies de Melipona como es el caso del trabajo realizado con propóleos de $M$. scutellaris contra $S$. aureus así como la cepa resistente a meticilina el cual resultó en una CMI de 6.25-12.5 mg mL-1 (Da Cunha et al., 2013). Para el caso de Scaptotrigona, se realizó un trabajo con abejas del género Trigona encontrándose con valores de CMI en el rango de 1.21 a $4.87 \mu \mathrm{g} \mathrm{mL}^{-1}$ contra tres cepas del S. aureus (Choudhari, Punekar, Ranade, \& Paknikar, 2012). Concentraciones menores en comparación con las CMI encontradas en este estudio.

El efecto antibacteriano de los propóleos evaluados en este estudio puede deberse a la vegetación en la zona de pecoreo de las abejas la cual influye tanto en la composición química así como la CMI de cada propóleos (Uzel, Önçağ, Çoğulu, \& Gençay, 2005). La actividad de los propóleos tanto en extractos acuosos como alcohólicos puede deberse a la disolución de los compuestos con actividad antimicrobiana así como la volatilidad la que facilita el contacto con la pared celular de las bacterias, logrando así la reducción de los factores de virulencia de estas cepas (Hazem et al., 2017). 
Se ha demostrado que los propóleos son capaces de inhibir enzimas como coagulasa y lipasa, los cuales facilitan a la bacteria patógena poder infectar al huésped (Sprin \& Bankova, 2011). Así mismo, los propóleos son capaces de actuar a nivel de $\mathrm{ADN}$, inhibiendo la replicación e indirectamente la división celular, evitando así la proliferación de la bacteria (Takaisi-Kikuni \& Schilcher, 1994).

Aunque existen datos sobre la actividad antimicrobiana de los propóleos de abejas sin aguijón, los resultados obtenidos pueden considerarse como los primeros datos reportados para $M$. solani y $S$. mexicana. Estos resultados se deben a que los propóleos han demostrado sinergismo entre sus componentes como se reporta en trabajos donde se analizaron tanto de forma integral, así como sus fracciones (Sforcin, Fernandes, Lopes, Bankova, \& Funari, 2000, Santos et al., 2002).

Algo similar ocurre con el ajo ya que el mecanismo de A. sativum se basa principalmente en la acción de la Alicina que actúa parcialmente a nivel de ADN inhibiendo la síntesis de proteínas e inhibiendo totalmente la síntesis de ARN como su principal objetivo (Eja et al., 2007). Para el caso de Allium sativum, La CMI se encuentra dentro del rango de valores reportados por otros autores que trabajaron tanto con extractos etanolicos como acuosos. Estos valores pueden llegar hasta los $75 \mathrm{mg} \mathrm{mL}^{-1}$ (Iwalokun, Ogunledun, Ogbolu, Bamiro, \& Jimi-Omojola, 2004, Betoni, Mantovani, Barbosa, Di Stasi, \& Fernandes, 2006, Abubakar, 2009). Sin embargo, la actividad antimicrobiana de $A$. sativum dependerá de la relación entre sus componentes bioactivos como la alicina y los sulfuros. Un estudio realizado con alicina liquida reportó que concentraciones bajas $\left(<62.5 \mu \mathrm{g} \mathrm{mL}^{-1}\right)$ de dicho compuesto, no mostró actividad antimicrobiana contra $S$. aureus; mientras que a $250 \mu \mathrm{g} \mathrm{mL}^{-1}$ o cercano a esta concentración fueron las más activas (Cutler \& Wilson, 2004). En el caso de los sulfuros, a mayor número de puentes disulfuros, mayor actividad antimicrobiana reflejan los compuestos. Un trabajo realizado con aceite de ajo reveló que una concentración alta de tetrasulfuro de dialilo puede conllevar a una mayor actividad antimicrobiana (Tsao \& Yin, 2001). Dichos trabajos realizados pueden dar dilucidar porqué $A$. sativum demostró una actividad antimicrobiana débil.

El análisis del índice de interacción mostró un efecto aditivo para los 3 propóleos de $S$. mexicana en combinación con A. sativum. Trabajos donde se ha combinado el propóleos con otras sustancias orgánicas como Lippia graveolens y el propio $A$. sativum han resultado en efectos sinérgicos $(\gamma<1)$ (Noori, Al-Ghamdi, Ansari, Al-Attal, \& Salom, 2012, Moreno et al., 2014). Aunque el resultado que se obtuvo no sea de carácter sinérgico, podemos decir que la acción individual del propóleos es igual de efectiva que en su combinación. Incluso podría utilizarse en cantidades menores a la $\mathrm{CE}_{5 \mathrm{O}}$ de ambos materiales vegetales. Por otro lado, también se han realizado trabajos de combinación del propóleos con antibióticos como Ciprofloxacino y Levofloxacino donde la actividad antibacterial se vio afectada (Lozano, López, Luis, Pacheco, \& Guillén, 2014). Algo similar ocurrió en nuestro estudio dado que la combinación del propóleos de M. solani y A. sativum dio como resultado un índice de interacción menor a la unidad (0.3). Una explicación a esto sería que el propóleos solo actúa de manera positiva con otros compuestos que actúen a nivel de pared celular y el mecanismo de acción del propóleos de M. solani sea distinto al de A. sativum o existan otros factores propios de dicho material que disminuyen su actividad al estar presente a los compuestos de A. sativum. Estas aseveraciones están basadas en la poca información que existe con base a la combinación del propóleos con otras sustancias orgánicas por lo que se requiere seguir investigando los mecanismos de acción en este tipo de estudios.

El efecto aditivo en las combinaciones demostró que de forma individual cada compuesto es igual de efectivo que en al realizar la combinación, y este representa un potencial para inhibir cepas de Staphylococcus aureus resistente a meticilina. 
Declaración de ética: los autores declaran que todos están de acuerdo con esta publicación y que han hecho aportes que justifican su autoría; que no hay conflicto de interés de ningún tipo; y que han cumplido con todos los requisitos y procedimientos éticos y legales pertinentes. Todas las fuentes de financiamiento se detallan plena y claramente en la sección de agradecimientos. El respectivo documento legal firmado se encuentra en los archivos de la revista.

\section{RESUMEN}

Introducción: El uso excesivo de antibióticos ha traído el aumento de resistencia en los microorganismos patógenos que provocan mortalidad de los pacientes. Por consiguiente, la búsqueda de nuevas estrategias naturales y no convencionales se ha vuelto constante e importante. Objetivo: Determinar la actividad antimicrobiana contra Staphylococcus aureus resistente a meticilina (SARM) de los propóleos por abejas sin aguijón. Métodos: Nueve fueron los extractos etanolicos de propóleos al $20 \%$ de las abejas sin aguijón de distintas regiones: de Melipona beecheii, de Melipona solani, de Tetragonisca angustula y de Scaptotrigona mexicana. Se realizó la caracterización química de los compuestos por cromatografía liquida y las pruebas de resistencia microbiana por el método de macrodilución para determinar el efecto de las combinaciones. Resultados: Los compuestos de interés fueron detectados, y destacan como los más abundantes los ácidos hidroxicinámicos, flavanonas, flavonoides y sus derivados glicosilados. Cuatro de los nueve propóleos resultaron efectivos contra SARM, los cuales provinieron, uno de Melipona solani y tres de Scaptotrigona mexicana. La CMI para $S$. mexicana está en el rango de $3-8 \mathrm{mg} \mathrm{mL}^{-1}$ y para $M$. solani fue de $4 \mathrm{mg} \mathrm{mL} \mathrm{mL}^{-1}$. Los estudios isobolográficos dieron como resultado un efecto aditivo $(\gamma=1)$ para la combinación de Allium sativum con los 3 propóleos de $S$. mexicana y un efecto antagónico $(\gamma>1)$ para la combinación de $A$. sativum con el propóleos de $M$. solani. Conclusiones: la combinación de extractos menores puede ser más efectiva que usando la CE50 de los extractos de forma individual. Se requieren estudios más detallados para definir los mecanismos de los propóleos de las abejas sin aguijón, así como su combinación con otras sustancias orgánicas.

Palabras clave: extractos; Meliponini; trigonas; Staphylococcus aureus; isobologramas; inhibición.

\section{REFERENCIAS}

Abubakar, E. (2009). Efficacy of crude extracts of garlic (Allium sativum Linn.) against nosocomial
Escherichia coli, Staphylococcus aureus, Streptococcus pneumoniea and Pseudomonas aeruginosa. Journal of Medicinal Plants Research, 3(4), 179-85.

Ahn, M., Kumazawa, S., Hamasaka, T., Bang, K., \& Nakayama, T. (2004). Antioxidant activity and constituents of propolis collected in various areas of Korea. Journal of Agricultural and Food Chemistry, 52(24), 7286-7292.

Amber, K., Aijaz, A., Immaculata, X., Luqman, K., \& Nikhat, M. (2010). Anticandidal effect of Ocimum sanctum essential oil and its synergy with fluconazole and ketoconazole. Phytomedicine, 17(12), 921-925.

Andersson, D., \& Hughes, D. (2011). Persistence of antibiotic resistance in bacterial populations. FEMS Microbiology Review, 35(5), 901-11.

Araújo, M., Búfalo, M., Conti, B., Fernandes, Jr. A., Trusheva, B., Bankova, V., \& Sforcin, J.M. (2015). The chemical composition and pharmacological activities of geopropolis produced by Melipona fasciculata Smith in Northeast Brazil. Journal of Molecular Pathophysiology, 4(1), 12-20.

Bankova, V., De Castro, S., \& Marcucci, M. (2000). Propolis: recent advances in chemistry and plant origin. Apidologie, 31(1), 3-15.

Betoni, J., Mantovani, R., Barbosa, L., Di Stasi, L., \& Fernandes, Jr. A. (2006). Synergism between plant extract and antimicrobial drugs used on Staphylococcus aureus diseases. Memórias do Instituto Oswaldo Cruz, 101(4), 387-90.

Bonvehí, J., \& Coll, F. (1994). Phenolic composition of propolis from China and from South America. Zeitschrift fur Naturforschung, 49(11-12), 712-718.

Boutabet, K., Kebsa, W., Alyane, M., \& Lahouel, M. (2011). Polyphenolic fraction of Algerian propolis protects rat kidney against acute oxidative stress induced by doxorubicin. Indian Journal of Nephrology, 21(2), 101-106.

Campos, J., dos Santos, U., Macorini, L., de Melo, A., Balestieri, J., Paredes-Gamero, E., Cardoso, C.A., \& dos Santos, E.L. (2014). Antimicrobial, antioxidant and cytotoxic activities of propolis from Melipona orbignyi (Hymenoptera, Apidae). Food and Chemical Toxicology, 65, 374-80.

Castro, M., Vilela, W., Zauli, R., Ikegaki, M., Rehder, V., Foglio, M., \& Rosalen, P.L. (2009). Bioassay guided purification of the antimicrobial fraction of a Brazilian propolis from Bahia state. BMC Complementary of Alternative Medicine, 9(1), 25-31.

Chanda, S., \& Rakholiya, K. (2011). Combination therapy: Synergism between natural plant extracts and antibiotics against infectious diseases. Microbiology Book Series, 1, 520-529. 
Choudhari, M., Haghniaz, R., Rajwade, J., \& Paknikar, K. (2013). Anticancer activity of Indian stingless bee propolis: an in vitro study. Journal of Evidence Based Complementary and Alternative Medicine, 2013, 1-10. DOI: $10.1155 / 2013 / 928280$

Choudhari, M., Punekar, S., Ranade, R., \& Paknikar, K. (2012). Antimicrobial activity of stingless bee (Trigo$n a \mathrm{sp}$.) propolis used in the folk medicine of Western Maharashtra, India. Journal of Ethnopharmacology, 141(1), 363-367.

Cutler, R., \& Wilson, P. (2004). Antibacterial activity of a new, stable, aqueous extract of allicin against methicillin-resistant Staphylococcus aureus. British Journal of Biomedical Science, 61(2), 71-74.

Da Cunha, M., Franchin, M., Galvão, L., de Ruiz, A., de Carvalho, J., Ikegaki, M., \& Rosalen, P.L. (2013). Antimicrobial and antiproliferative activities of stingless bee Melipona scutellaris geopropolis. BMC Complementary and Alternative Medicine, 13(1), 23-32.

De Castro, S. (2001). Propolis: biological and pharmacological activities. Therapeutic uses of this bee-product. Annual Review of Biomedical Sciences, 3, 49-83.

Dos Santos, P.A., Bicalho, B., \& de Aquino Neto, F. (2003). Comparison of propolis from Apis mellifera and Tetragonisca angustula. Apidologie, 34(3), 291-298.

Fernandes, Jr. A., Leomil, L., Fernandes, A., \& Sforcin, J. (2001). The antibacterial activity of propolis produced by Apis mellifera L. and Brazilian stingless bees. Journal of Venomous Animals and Toxins, $7(2), 173-182$

Eja, M., Asikong, B., Abriba, C., Arikpo, G., Anwan, E., \& Enyi-Idoh, K. (2007). A comparative assessment of the antimicrobial effects of garlic (Allium sativum) and antibiotics on diarrheagenic organisms. The Southeast Asian Journal of Tropical Medicine in Public Health, 38(2), 343-348.

Guillamón, E. (2018). Efecto de compuestos fitoquímicos del género Allium sobre el Sistema immune y la respuesta inflamatoria. Ars Pharmaceutica, 59(3), 185-196.

Hazem, A., Popescu, C., Crisan, J., Popa, M., Chifiriuc, M., Pircalabioru, G., \& Lupuliasa, D. (2017). Antibacterial efficiency of five propolis extracts on planktonic and adherent microbial strains. Farmacia, 65(5), 813-818.

Huang, S., Zhang, C., Wang, K., Li, G., \& Hu, F. (2014). Recent advances in the chemical composition of propolis. Molecules, 19(12), 19610-19632.

Inui, S., Hatano, A., Yoshino, M., Hosoya, T., Shimamura, Y., Masuda, S., \& Kumazawa, S. (2014). Identification of the phenolic compounds contributing to antibacterial activity in ethanol extracts of
Brazilian red propolis. Natural Product Research, 28(16), 1293-1296.

Iwalokun, B., Ogunledun, A., Ogbolu, D., Bamiro, S., \& Jimi-Omojola, J. (2004). In vitro antimicrobial properties of aqueous garlic extract against multidrugresistant bacteria and Candida species from Nigeria. Journal of Medicinal Food, 7(3), 327-333.

Kumazawa, S., Hamasaka, T., \& Nakayama, T. (2004). Antioxidant activity of propolis of various geographic origins. Food Chemistry, 84(3), 329-339.

Lotti, C., Campo-Fernandez, M., Piccinelli, A., CuestaRubio, O., Marquez-Hernandez, I., \& Rastrelli, L. (2010). Chemical constituents of red Mexican propolis. Journal of Agricultural and Food Chemical, 58(4), 2209-2213.

Lozano, E., López, O., Bocanegra, M., Davis, L., Flores, C., \& Cervantes, M. (2013) Interacción sinérgica de propóleos (Propolis) y orégano (Lippia graveolens Kunth sl) contra Staphylococcus aureus. Revista Mexicana de Ciencias Farmacéuticas, 44(4), 73-78.

Lozano, E., López, O., Luis, A., Pacheco, J., \& Guillén, A. (2014). Interaction between propoleum extracts and Ciprofloxacin and Levofloxacin for the in vitro inhibition of methicillin-resistant Staphylococcus aureus isolates. African Journal of Microbiology Research, 8(10), 1089-1097.

Marcucci, M., Ferreres, F., García-Viguera, C., Bankova, V., De Castro, S., Dantas, A., \& Paulino, N. (2001). Phenolic compounds from Brazilian propolis with pharmacological activities. Journal of Ethnopharmacology, 74(2), 105-112.

Massaro, C., Simpson, J., Powell, D., \& Brooks, P. (2015). Chemical composition and antimicrobial activity of honeybee (Apis mellifera ligustica) propolis from subtropical eastern Australia. The Science of Nature, 102(68), 11-12.

Miorin, P., Levy Junior, N., Custodio, A., Bretz, W., \& Marcucci, M. (2003). Antibacterial activity of honey and propolis from Apis mellifera and Tetragonisca angustula against Staphylococcus aureus. Journal of Applied Microbiology, 95(5), 913-920.

Moreno-Cruz, F., Cervantes-Flores, M., Lopez-Guzman, O., Vertiz-Hernández, A., Ceniceros-Medina, R., \& Lozano-Guzman, E. (2014). Synergistic interaction of extracts of garlic (Allium sativum) and propolis against methicillin-resistant Staphylococcus aureus. African Journal of Microbiology Research, 8(52), 3986-3991.

Morroni, G., Álvarez-Suarez, J., Brenciani, A., Simoni, S., Fioriti, S., Pugnaloni, A., ... \& Giovanetti, E. (2018). Comparison of the antimicrobial activities of four honeys from three countries (New Zeeland, Cuba, and Kenya). Frontiers in Microbiology, 9, 1378-1387. 
Nina, N., Quispe, C., Jiménez-Aspee, F., Theoduloz, C., Feresín, G.E., Lima, B., ... \& Schmeda, H.G. (2015). Antibacterial activity, antioxidant effect and chemical composition of propolis from the Región del Maule, Central Chile. Molecules, 20(10), 18144-18167.

Noori, A., Al-Ghamdi, A., Ansari, M., Al-Attal, Y., \& Salom, K. (2012). Synergistic effects of honey and propolis toward drug multi-resistant Staphylococcus aureus, Escherichia coli and Candida albicans isolates in single and polymicrobial cultures. International Journal of Medical Science, 9(9), 793-800.

Popova, M., Dimitrova, R., Al-Lawati, H., Tsvetkova, I., Najdenski, H., \& Bankova, V. (2013). Omani propolis: chemical profiling, antibacterial activity and new propolis plant sources. Chemistry Central Journal, $7(1), 1-8$

Popova, M., Chinou, I., Marekov, I., \& Bankova, V. (2009). Terpenes with antimicrobial activity from Cretan propolis. Phytochemistry, 70(10), 1262-1271.

Rajic-Savic, N., \& Valovic, J. (2009). Antimicrobial activity of propolis on the basis of preparation in relation to Staphylococcus aureus. In The international symposium on biocides in public health and environment [and] the international symposium on antisepsis, disinfection and sterilization [and] Belgrade (205-206). Serbia: Institute for Biocides and Medical Ecology.

Ristivojević, P., Dimkić, I., Trifković, J., Berić, T., Vovk, I., Milojković-Opsenica, D., \& Slavisa, S. (2016). Antimicrobial activity of Serbian propolis evaluated by means of MIC, HPTLC, bioautography and chemometrics. PloS one, 11(6), e0157097.

Salomão, K., Dantas, A., Borba, C., Campos, L., Machado, D., Aquino, N.F., \& De Castro, S.L. (2004). Chemical composition and microbicidal activity of extracts from Brazilian and Bulgarian propolis. Letters in Applied Microbiology, 38(2), 87-92.

Santos, F., Bastos, E., Uzeda, M., Carvalho, M., Farias, L., Moreira, E., \& Braga, F.C. (2002). Antibacterial activity of Brazilian propolis and fractions against oral anaerobic bacteria. Journal of Ethnopharmaco$\log , 80(1), 1-7$.

Santos, H., Campos, J., Santos, C., Balestieri, J., Silva, D., Carollo, C., ... \& Dos, S.E.L. (2017). Chemical profile and antioxidant, anti-inflammatory, antimutagenic and antimicrobial activities of geopropolis from the stingless bee Melipona orbignyi. International Journal of Molecular Science, 18(5), 953-971.

Sartori, G., Pesarico, A., Pinton, S., Dobrachinski, F., Roman, S., Pauletto, F., ... \& Prigol, M. (2012). Protective effect of brown Brazilian propolis against acute vaginal lesions caused by herpes simplex virus type 2 in mice: involvement of antioxidant and anti-inflammatory mechanisms. Cell Biochemistry and Function, 30(1), 1-10.

Sawaya, A., Souza, K., Marcucci, M., Cunha, I., \& Shimizu, M. (2004). Analysis of the composition of Brazilian propolis extracts by chromatography and evaluation of their in vitro activity against grampositive bacteria. Brazilian Journal Microbiology, 35(1-2), 104-109.

Serra, V.M.A. (2017). La Resistencia microbiana en el context actual y la importancia del conocimiento y aplicación en la política antimicrobiana. Revista Habanera de Ciencias Médicas, 16(3), 402-419.

Sforcin, J., Fernandes, Jr. A., Lopes, C., Bankova, V., \& Funari, S. (2000). Seasonal effect on Brazilian propolis antibacterial activity. Journal of Ethnopharmacology, 73(1-2), 243-249.

Shrestha, S., Narukawa, Y., \& Takeda, T. (2007). Chemical constituents of Nepalese propolis (II). Chemical and Pharmaceutical Bulletin, 55(6), 926-929.

Silici, S., \& Kutluca, S. (2005). Chemical composition and antibacterial activity of propolis collected by three different races of honeybees in the same region. Journal of Ethnopharmacology, 99(1), 69-73.

Soto, G., Moreno, L., \& Pahua, D. (2016). Panorama epidemiológico de México, principales causas de morbilidad y mortalidad. Revista de la Facultad de Medicina (México), 59(6), 8-22.

Sprin, J., \& Bankova, V. (2011) Propolis: is there a potential for the development of new drugs? Journal of Ethnopharmacoly, 133(2), 253-260.

Takaisi-Kikuni, N., \& Schilcher, H. (1994). Electron microscopic and microcalorimetric investigations of the possible mechanism of the antibacterial action of a defined propolis provenance. Planta Medica, 60(3), 222-227.

Tallarida, R.J. (2001). Drug synergism: its detection and applications. Journal of Pharmacology and Experimental Therapeutics, 298(3), 865-872.

Taroco, R., Seija, V., \& Vignoli R. (2006). Métodos de estudio de sensibilidad antibiótica. Sección I, Control de poblaciones microbianas. Temas de Bacteriología y Virología Médica. Montevideo, Uruguay: Universidad de la República.

Trusheva, B., Todorov, I., Ninova, M., Najdenski, H., Daneshmand, A., \& Bankova, V. (2010). Antibacterial mono-and sesquiterpene esters of benzoic acids from Iranian propolis. Chemistry Central Journal, $4(1), 8-12$.

Tsao, S.M., \& Yin, M.C. (2001). In-vitro antimicrobial activity of four diallyl sulphides occurring naturally 
in garlic and Chinese leek oils. Journal of Medical Microbiology, 50(7), 646-649.

Ushimaru, P., Barbosa, L., Fernandez, A., Di Stasi, L., \& Júnior, A. (2012). In vitro antibacterial activity of medicinal plant extracts against Escherichia coli strains from human clinical specimens and interactions with antimicrobial drugs. Natural Product Research, 26(16), 1553-1557.
Uzel, A., Önçağ, Ö., Çoğulu, D., \& Gençay, Ö. (2005). Chemical compositions and antimicrobial activities of four different Anatolian propolis samples. Microbiological Research, 160(2), 189-195.

Wang, K., Zhang, J., Ping, S., Ma, Q., Chen, X., Xuan, H., ... \& Hu, F. (2014). Anti-inflammatory effects of ethanol extracts of Chinese propolis and buds from poplar (Populus $\times$ canadensis). Journal of Ethnopharmacology, 155(1), 300-311. 